\title{
Non-pharmaceutical Therapy for Depression
}

\author{
Ruotong $\mathrm{Xu}^{1}$ \\ ${ }^{1}$ The Second-high School Attached to Beijing Normal University, Beijing 102600, China
}

\begin{abstract}
At present, the non-drug treatment of depression has developed rapidly. Diverse methods which have quick effects, less injury, good adherence and other advantages are becoming available for all kinds of people. However, clinical workers need to choose treatment for patients according to their individual characteristics with an accurate and flexible use of non-drug therapy approach, in order to quickly and effectively reduce the patients' pain, allow their souls to return home and return to society. Nevertheless, it still needs to be further explored and studied. This article discusses the treatment of non-medicated depression by looking up documents online about non-pharmaceutical treatment of depression to help people find the best way to get rid of the disease without relying partly on drugs and to provide references for medical treatment.
\end{abstract}

\section{Introduction}

With the quickening pace of life and increasing competition in society, more and more people are bombarded with various assignments as pressure raises rapidly. People lost their motivation and became stressful thus depression occurs. Depression is one of the most common symptoms around world suffered by over 90 million people from different countries in different levels, which are disorders including feeling, sleeping and daily activities of people. The incidence of depression is increasing year by year. According to the World Health Organization, depression will become the second largest burden of disease by 2020 . There are $8.7 \%$ and $5.3 \%$ of women and men getting depressed. And with their age level increase, the proportion of getting depressed raises too $[8]$.

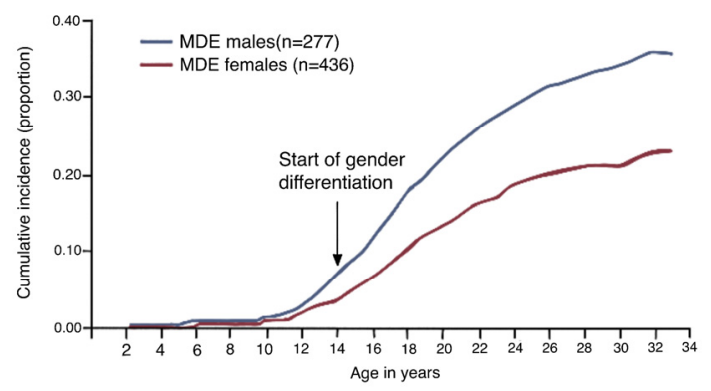

Fig. 1. Age of onset hazard ratios for major depressive episode (MDE) in males and females [10]

What is more, among Chinese children who aged 8-16 years old, $34.57 \%$ of them are suffering from depression [1]. This paper will talk about the non-pharmaceutical therapy of depression from four aspects. For starters, elements contribute to depression and its clinical manifestation. Elements could be divided into the genetic inheritance which is innate and mostly has to do with their family genes, as well as the environmental factor that would trigger the symptom of depression. To study the non-pharmaceutical therapy for treating depression, people need to know different types of depressions and their clinical manifestation from both physical and mental actions, which will also be mentioned in the following text. Secondly, this paper will talk about some of the non-pharmaceutical therapies and the psychotherapy. In order to analyse and provide suggestions for them, the paper will include the discussion of the advantages and drawbacks about drug and non-drug uses for treatment. The third part is to investigate the prospects of non-drug therapy and the overall tendency about treatment of depression. The purposes of the paper through studying the psychotherapy for depression are to give suggestions to a better treatment decision and to encourage people not to totally rely on drugs for remedying depression.

\section{Symptoms of Depression}

Depression has a sort of symptoms, including feelings of guilt, hopelessness and loneliness as well as being difficult to concentrate or remember details. Some of depressions may only occur during the day or night. The reason for it has to do with many subjects such as biochemistry, neuroimmunology, social psychology. Although there has not been any clear answer to the formation of depression, there do have some fields that contribute to it. The genetic factor means the expression of parental traits in offspring. Depression has a family clustering phenomenon, the closer the blood relationship is, the higher the probability of depression will be. There

\footnotetext{
*Corresponding author: mali@shsbnu.cn
} 
are certain kinds of comparative genetic studies. The first type is kinship, and it means that relatives of people with depression are far more likely to suffer from it, and they are about 10 to 30 times more likely to suffer from depression than the general population, which means those children who were born to depressed parents are significantly more likely to be depressed than children born to normal parents. What is more, if one parent is depressed, the child's chance of becoming depressed increases by $10-13$ percent. And if both of the parents develop the disease, the offspring may have up to $50 \%$ of the cases. For twins, according to the report of 10 scholars from 1928 to 1977,155 out of 232 pairs of identical twins suffered from depression, which was the same rate of $66.8 \%$. Of the 435 pairs of fraternal twins, 68 had the same depression, with a concordant rate of $15.6 \%$. It can be seen that the rate of identical twins is significantly higher than that of fraternal twins. And for foster child, the observation of twins reared apart in different environments after birth is called the study of foster children. To separate the effects of genetics from upbringing, the researchers looked at how often children of depressed parents were adopted by normal parents. In 1968, Presse looked at the concordant rates of depression among identical twins reared apart or not reared apart after birth and found that the concordant rates of depression were $65 \%$ among identical twins reared apart. The incidence of the same disease without separate upbringing was $68 \%$, and the environmental factor took up $3 \%$ [2].

However, for the exact reasons of depression, there is no complete understanding of it. People who suffer from depression may have had experiences of failure and frustration, losing parents or getting severe diseases. There are some elements that might be related to it. The way that how stress causes depression depends on how people regard it. The proper amount of stress can act as a stimulant. For example, when people regard examinations to a sort of challenge, stress will definitely have a positive effect on human since the stress hormones will not have a huge negative impact on their body. Nevertheless, stress is like diseases which are long-term problems and unchangeable in short time but could easily cause depression, e.g. poverty. The next important factor is that different ways of thinking can also lead to different levels of depression. False perceptions of events and exaggerated thoughts of consequences could have a strong bond with depression by forcing brain to enter a negative situation and eventually become hopeless with themselves. From the physical aspect, those kinds of passive thinking style will exaggerate the response to painful stimulation and increase the behavioral response of the stress hormone cortisol and pro-inflammatory cytokines in the blood [3].

\section{Treatments of Depression}

\subsection{Medical Treatment}

In order to put depression down, people have to get the treatment that works best for themselves with the least side effects. Thus, according to different circumstances, various people should consider different ways for treatment. There are two types of treatment that can help people get rid of depression or at least reduce the level of it. The first one is medical treatment which does have a lot of side effects. And the second method is the non-pharmaceutical treatment. As for the former one, there is a drug called antidepressants which is used all around the world today, serving as the first choice for depression treatment.

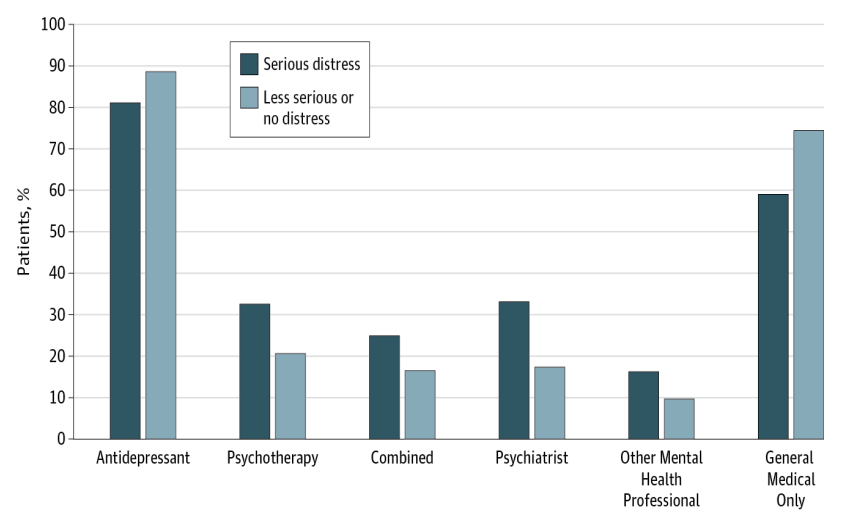

Fig.2. Treatments for depression for adults [12]

When people fall into depression, the brain circuits where norepinephrine, serotonin and dopamine are located in, are not working in an appropriate way. And the antidepressants could help the brain work back on track [4]. The types of antidepressants are diverse, and there are different drugs that have various side effects [7]. The common side effects include headaches, anxiety, gaining weight and other abnormal reactions. However, for different people, they may have varying degrees of reactions. For example, it is very common that when two patients are using the same drug to treat the same level of depression, one of them has no problem with the side effects, but the other has more than one serious reaction. Therefore, choosing the way for treating depression has connections with different situation of individual's body.

\subsection{Non-pharmaceutical Treatment}

As for the latter kind of treatment, there are many approaches that have been invented. At present, there are several kinds of ways are available.

\subsubsection{MECT}

The modified electroconvulsive therapy, MECT [5]. Currently, MECT is widely used in psychiatric clinical practice as it has the high-safety index with no absolute contraindication. It is also commonly used in the treatment of severe and refractory depression. Its efficiency is higher with the rate of $80-90 \%$ compared to drug therapy. Relevant experiments have shown that the metabolism of the local areas of the brain of patients will be continuously enhanced after MECT treatment, and the neuro factors that can improve mental symptoms and 
mood will also be partially increased. The best way is to combine this therapy with relevant drugs that was recommended by therapist to patients according to their own situation in order to reach the best result. In terms of adverse reactions, both groups of patients had mild adverse reactions, which were relieved after appropriate treatment. The research results of other scholars have also confirmed that the therapeutic effect of drug combination with MECT is superior to that of drug therapy alone, and domestic studies on the therapeutic effect and safety of MECT are relatively higher. At the end of MECT treatment, patients' memory was reduced to a certain extent, but this reduction was reversible. One month after finishing the treatment, the reduced memory could be restored to the pre-treatment level; and compared with the pre-treatment, patients' MMSE (mini-mental State Examination) score was improved to a certain extent. Foreign studies have also confirmed that MECT does not damage the high-level cognitive function of patients, but only slows down the recipient's information processing speed to some extent. To sum up, MECT is characterized by rapid onset, accurate efficacy and high safety. Currently, it is widely used in clinical practice, and the technology is relatively mature, which can significantly improve the treatment compliance.

\subsubsection{TMS}

The transcranial magnetic stimulation treatment, TMS, which is a non-invasive treatment that affects many metabolism and electrical activities in the brain, through depolarization and hyperpolarization of neurons in brain tissue [9]. Some studies have also confirmed that the insufficiency of the left prefrontal cortex of the brain can cause depression. Transcranial magnetic stimulation affects nerve cells through repeated stimulation of the DLPFC region of the brain, thus reducing the onset of depression. With its high safety, simple operation, and the advantages of non-invasive treatment, it has been widely used in clinical psychiatry.

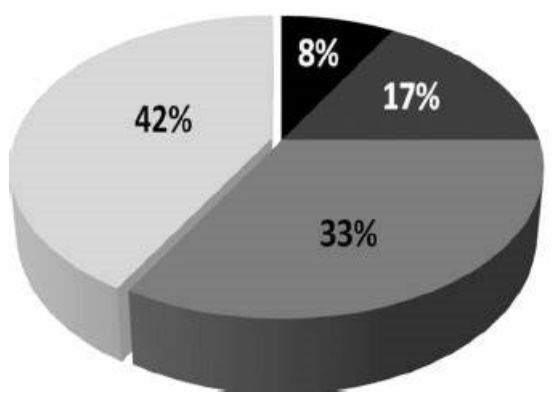

\section{Percentage of} improvement after TMS
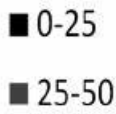

Fig. 3. Improvement of patients with TMS [11]

Although the mechanism of TMS is still unclear, it is generally believed that TMS can improve patients' brain function and metabolism, increase local cerebral blood flow, improve excitability of cerebral cortex, and ultimately promote neurotransmitter changes in the brain by generating transient magnetic fields and acting on brain tissues. The rTMS is especially useful for patients who have suicidal tendency because of its short course of treatment [6].

\subsubsection{Psychotherapy}

The next part is about psychotherapy. Cognitive and behavioral therapy contains many therapies like psychoanalytic therapy, behavioral therapy, cognitive therapy, marriage and family therapy, morita therapy, which are operated through the application of relevant techniques and theories. Professional psychotherapists help clients to alleviate or eliminate psychological symptoms and problems and promote the improvement of potential and motivation of visitors. Those kinds of treatments do not have many side effects. However, it should be noted that both cognitive and cognitive behavioral therapy require higher requirements of the therapist. If the therapist has limited experience, it is difficult for patients to obtain high-quality treatment, and relevant talents need to be extensively cultivated.

\section{Other Treatments}

\subsection{Art Therapy}

In addition to all these methods, there are many other treatments. The art therapy like painting therapy, which tends to be a kind of unconscious communication that makes the whole process of the treatment appear more natural and smoother so that patients could express their subconscious thoughts and feelings in the form of pictures. Through painting therapy, patients can judge themselves, understand their own psychological state, and release their emotions unconsciously, thus achieving the purpose of treatment. Many domestic studies have also shown that painting therapy can significantly improve the mood disorders of patients with mildness to moderate depression.

\subsection{Sand Treatment}

Besides, sand table therapy is game to guide patients to make free creation with sand, water and models with the help of sand table. It is not only conducive to combing patients' psychological problems and clearing up their thoughts, but also helps to promote the expression of their subconscious consciousness and assists them to know themselves, so as to finally achieve the purpose of treatment. In foreign countries, sandbox treatment has been very mature, and there are many researchers reported that have confirmed the effect of sandbox treatment. Sandbox therapy was introduced into China in the 1990s, and there has been a wide range of clinical experience.

\subsection{Relaxation Therapy}

What is more, relaxation therapy is a kind of psychological therapy to control people's own psycho-physiological activities by consciously training to reduce the level of arousal and improve the body function disorders. For instance, exercising and traveling from old place to a new place periodically could also 
help people get out of depression when the bad mood or feelings are not deeply rooted in mind. Depression caused by uncomfortable atmosphere could be eliminated in a new environment by getting away from the oppressive circumstances.

\section{Conclusion}

In conclusion, depression should be treated in different ways according to the order severity of patient. If it is a mild depression, it can be treated and improved by non-medical means which does not have a lot of side effect compared to drug therapy, such as regular sleep and rest, a balanced diet, regular exercise and increased outdoor activities, psychological diary, reading and art therapy as mentioned above. For patients with moderate or severe depression, drug treatment is necessary, it is recommended to standardize the diagnostic evaluation institutions, otherwise severe depression is prone to self-suicide and other serious consequences. In order to achieve the best effect of treatment, the combination of drugs and non-drugs should be used to treat depression together according to the patient's own situation.

In writing this paper, although the author has read a lot of literature, this research still has some shortcomings. There is still a number of things like the VNS, which is also one of the non-pharmaceutical treatment and other factors that are not covered in the paper. Therefore, the author will be continuing working on. In the future, research might be conducted on the relationship between depression and the brain, which will be a further study.

In the process of writing this paper, many people gave me great help and support. I would like to express my thanks respectively. Firstly, Mr. Dalley, my biology professor, who gave me the lesson of studies on the psychological mechanism of brain dysfunction which helped me writing this article in the academic aspect. Secondly, my mother, who supports me all the time, for whatever she thinks is right and good for me. Thirdly, my advisors and teaching assistant, who helped me gaining that knowledge and writing a better essay. Last but not the least, my friends who supplied me with a lot of relevant information and helped with the process of posting an essay.

\section{References}

1. A. Morin, LCSW, Depression Statistics Everyone Should Know (2020), online available https://www.verywellmind.com/depression-statistics -everyone-should-know-4159056, accessed on November 10th, 2020.

2. Psychology Today, What are the most common causes of depression (2020), online available https://www.psychologytoday.com/us/basics/depress ion/causes-depression\#does-depression-have-some-h idden-trigger, accessed on November 9th, 2020.

3. WebMD, Depression treatment of antidepressant drugs (2016), online available https://www.webmd.com/depression/guide/depressio n-treatment-options\#1-2, accessed on November 7th, 2020 .

4. X. Y. Luan and X. M. Hu, Present situation of non-pharmacotherapy for depression Journal of Clinical Psychosomatic Diseases, 2 (2015)

5. L. Q. Zhu, H. Bo DR, What are the non-drug treatments for depression? (2018), online available https://m.baidu.com/bh/m/detail/qr_9772213387932 409281, accessed on November 8th, 2020.

6. G. J. Xu, Z. Song and J. Y. Wang, Advances in research on non-drug therapy for depression, Chinese Practical Journal of Rural Doctor, 24, 9, (2017)

7. A. Bjarnadottir, healthline, A guide to common antidepressant side effects (2018), online available https://www.healthline.com/health/antidepressant-si de-effects, accessed on November 10th, 2020.

8. SoHu, 2018, General situation of depression in China, online available https://www.sohu.com/a/275994291_100281680, accessed on November 9th, 2020.

9. L. Liu, H. Dai, Z. X. Fu, How does depression affect brain structure (2017), online available https://mip.haodf.com/zhuanjiaguandian/522562540 0.htm, accessed on November 10th, 2020.

10. K. Beesdo, M. Höfler, E. Leibenluft, R. Lieb, M. Bauer, A. Pfennig (2009), Bipolar Disord. online available

https://www.medicographia.com/2010/10/the-timing -of-depression-an-epidemiological-perspective/ accessed on November 10th, 2020.

11. B. Degos, et al. Transcranial magnetic stimulation as an efficient treatment for psychogenic movement disorders, Journal of Neurology, Neurosurgery, and Psychiatry, February 2013, online available https://www.researchgate.net/figure/Main-clinical-ch aracteristics-of-the-24-patients-included-in-the-study tbl1_235405623, accessed on November 10th, 2020.

12. M. Olfson, C. Blanco, Steven C. Marcus, JAMA Network, Treatment of Adult Depression in the United States, JAMA Intern Med. 176, 10, pp.1482-1491 (2016). 